\title{
Photoluminescent gold nanomaterials as sensitive probes
}

\section{Editorial}

Photoluminescent gold nanomaterials have become interesting sensing materials because of their ease in preparation and conjugation, large Stokes shift, long lifetime, and biocompatibility. ${ }^{1-3}$ They are often called gold nanodots (Au NDs) or gold nanoclusters (Ag NCs), with sizes usually smaller than $2 \mathrm{~nm}$; Au NCs are commonly reffered to the one having less than $30 \mathrm{Au}$ atoms per cluster. The molecularlike optical properties (photoluminescence) of Au NDs and NCs are highly dependent on the size of $\mathrm{Au}$ core and the number of $\mathrm{Au}$ atoms per templates, respectively. ${ }^{4,5}$ The Au complexe on the surface of $\mathrm{Au}$ core and its surface density affect the stability and optical properties of $\mathrm{Au}$ NDs, while the nature and size of the template affect that of $\mathrm{Au}$ NCs. Thus, the optical properties of AuNDs/NCs can be easily tuned by selecting suitable templates or ligands and controlling their molar concentration ratio to $\mathrm{Au}^{3+}$. Their emission wavelengths are usually longer than $600 \mathrm{~nm}$ when excited with a UV light (commonly at $365 \mathrm{~nm}$ ). With a support of having a large Stoke shift and long lifetime, it is believed that their photoluminescen is mainly through the ligand-metal charge transfer transition (LMCT) or ligand-tometal-metal charge transfer transition (LMMCT).

Au NDs can be prepared from etching of small Au nanoparticles $(<3$ $\mathrm{nm}$ in diameter) by thiol compounds such as 11-mercaptoundecanoic acid (11-MUA) under alkaline conditions. ${ }^{6}$ On the other hand, proteins such as bovine serum albumin (BSA), lysozyme, and horseradish peroxidase as a template and reducing agent are commonly used to prepare stable $\mathrm{Au} \mathrm{NCs}{ }^{7}$ The reducing ability of these proteins mainly comes from their tyrosine residues that can reduce $\mathrm{Au}^{3+}$ at $\mathrm{pH}$ values $>10.0$. Although $\mathrm{Au}$ NCs can also be prepared using thiol compounds such as glutathione as a reducing agent, they are usually less stable and have weaker photoluminescence intensity than that of BSA-Au NCs. Protein-Au NCs are more stable than 11-MUA -Au NDs against photo irradiation and salt induced quenching, mainly because large protein molecule protect $\mathrm{Au}$ core more efficiently, which minimizes the access of quenchers such as oxygen to the core. While, large amounts (usually $>$ several $\mathrm{mM}$ ) of expensive proteins are required to prepare stable and bright $\mathrm{Au}$ NCs.

$\mathrm{Au}$ NDs and NCs have been employed for sensing of various analytes such as metal ions, anions and proteins, as well as for cell imaging. 11-MUA-Au NDs were used for quantitation of $\mathrm{Hg}^{2+}$ using 2,6-pyridinedicarboxylic acid (PDCA) as a masking agent to prevent the interference from $\mathrm{Pb}^{2+}$ and $\mathrm{Cd}^{2+}$, with limit of detection (LOD) $5 \mathrm{nM}^{6}$ In the presence of $\mathrm{Hg}^{2+}$, photoluminescence quenching of 11-MUA-Au NDs occurs, due to the $\mathrm{Au}-\mathrm{Hg}$ aurophilic interaction and aggragation of the $\mathrm{Au}$ NDs through the interaction of $\mathrm{Hg}^{2+}$ ions with the carboxylate groups present on the surfaces of the 11-MUA-Au NDs. To minimize salt quenching, poly(N-isopropylacrylamide) microgels (PNIPAM MGs) containing 11-MUA-Au NDs were prepared and used for quantitation of $\mathrm{Hg}^{2+}$ in the presence of $500 \mathrm{mM} \mathrm{NaCl}$, with LOD of $1.7 \mathrm{nM}^{8}$ Because $\mathrm{H}_{2} \mathrm{O}_{2}$ can oxidize the Au core of 11-MUA-Au NDs, leading to photoluminescence quenching, the Au NDs is selective for quantitation of glucose when using glucose oxidase to catalyze the

\author{
Volume 2 Issue 4 - 2017 \\ Jinshun Cang,' Huan-Tsung Chang'2,3 \\ 'Department of Chemical Engineering, Yancheng Institute of \\ Industry Technology, China \\ ${ }^{2}$ Department of Chemistry, National Taiwan University, Taiwan \\ ${ }^{3}$ Department of Chemistry, Chung Yuan Christian University, \\ Taiwan
}

Correspondence: Huan Tsung Chang, Department of Chemistry, National Taiwan University, Taipei, 10617, Taiwan, Email changht@ntu.edu.tw

Received: April 28, 2017 | Published: May 01, 2017

reaction of glucose and oxygen. ${ }^{9}$ 11-MUA-Au NDs conjugated with platelet-derived growth factors (PDGF) were used for quantitation of PDGF receptor, with great selectivity and sensitivity (LOD of $0.25 \mathrm{nM}) .{ }^{10} \mathrm{Au}$ NDs prepared from Au nanoparticles using different etching agents have been used for quantiation of various analytes, including $\mathrm{S}^{2-}, \mathrm{NO}_{2}^{-}$, concanavalin $\mathrm{A}($ Con $A)$, Phospholipase $\mathrm{C}$, and so on. ${ }^{11-14}$ Based on analyte-induced PL quenching, lysozyme-Au NCs were used for quantitation of $\mathrm{Hg}^{2+}$, with LODs of 10nM. ${ }^{15}$

Through core-etching induced PL quenching, BSA-Au NCs were used for the detection of cyanide with a LOD of $200 \mathrm{nM} .{ }^{16}$ BSA-Au NCs were used for determination of the activity of trypsin, with LOD of $86 \mathrm{pM} .{ }^{17}$ Through digestion of BSA by trypsin, the BSA shell was destroyed, leading to PL quenching as a result of oxidation of $\mathrm{Au}$ NCs by oxygen. To specifically target cancer cells, BSA-Au NCs can be conjugated with recognition elements such as small ligands and antibodies. For example, folic acid-BSA-Au NCs are specific toward cancer cells over-expressed folate receptors. ${ }^{18}$ A ratiometric probe using BSA-Au/Ce NCs was developed for monitoring the $\mathrm{pH}$ values of HeLa cells. ${ }^{19}$ The BSA-Au/Ce NCs possess dual emission bands at 410 and $650 \mathrm{~nm}$ when excited at $325 \mathrm{~nm}$, which separately correspond to the BSA-Ce complexes and Au NCs. The PL at $410 \mathrm{~nm}$ is pHdependent while that at $650 \mathrm{~nm}$ is $\mathrm{pH}$-insensitive. Because the red fluorescence of BSA-Au/Ce NCs is quenched by $\mathrm{H}_{2} \mathrm{O}_{2}$, the NCs with glucose oxidase were used for quantitation of glucose. ${ }^{20}$

Although biocompatible $\mathrm{Au}$ NCs and NDs have shown their potential for sensing of various analytes and for cell imaging, their quantum yields are generally lower than that of quantum dots ${ }^{21}$ and carbon dots. ${ }^{22}$ To provide specifiity toward tumors, strategies for improved stability and affinity through functionaliztion of Au NDs and NCs with biopolymers, synthetic poymers, small ligands are still highly demanded. It is also interesting to prepare theranostic nanomaterials (therapeutic drugs and diagnostic agents into one single platform) using other reporters and drugs to conjugate AuNCs/NDs. ${ }^{23}$ With advances in nanotechnology, it is our belief that more stable and brighter $\mathrm{Au} \mathrm{NCs} / \mathrm{NDs}$ will soon be realized. They may have great potential for cell tracking and for single molecule detection. 


\section{Acknowledgements}

This study was supported by the Ministry of Science and Technology (MOST) of Taiwan under contracts MOST 103-2923-M-002-002MY3 and 104-2113-M-002-008-MY3.

\section{Conflict of interest}

The author declares no conflict of interest.

\section{References}

1. Chen LY, Wang CW, Yuan Z, et al. Fluorescent gold nanoclusters: recent advances in sensing and imaging. Anal Chem. 2015;87(1):216-229.

2. Chen PC, Periasamy AP, Harroun SG, et al. Photoluminescence sensing systems based on copper, gold and silver nanomaterials. Coord Chem Rev. 2016;320:129-138.

3. Ravindranath R, Prathik R, Chang HT. Synthesis, optical properties, and sensing applications of gold nanodots. Chem Rec. 2016;16(3):16641675 .

4. Forward JM, Bohmann D, Fackler JP, et al. Luminescence studies of gold(I) thiolate complexes. Inorg Chem. 1995;34(25):6330-6336.

5. Luo Z, Yuan X, Yu Y, et al. From aggregation-induced emission of Au(I)thiolate complexes to ultrabright $\mathrm{Au}(0) @ \mathrm{Au}(\mathrm{I})$-Thiolate Core-Shell Nanoclusters. J Am Chem Soc. 2012;134(40):16662-16670.

6. Huang CC, Yang Z, Lee KH, et al. Synthesis of highly fluorescent gold nanoparticles for sensing mercury(II). Angew Chem Int Ed. 2007;46(36):6824-6828.

7. Kawasaki H, Hamaguchi K, Osaka I, et al. pH-dependent synthesis of pepsin-mediated gold nanoclusters with blue green and red fluorescent emission. Adv Funct Mater. 2011;21(18):3508-3515.

8. Chen LY, Ou CM, Chen WY, et al. Synthesis of photoluminescent Au ND-PNIPAM hybrid microgel for the detection of $\mathrm{Hg}^{2+}$. ACS Appl Mater Interfaces. 2013;5(10):4383-4388.

9. Shiang YC, Huang CC, Chang HT. Gold nanodot-based luminescent sensor for the detection of hydrogen peroxide and glucose. Chem Commun. 2009;23:3437-3439.

10. Huang CC, Chiang $\mathrm{CK}$, Lin $\mathrm{ZH}$, et al. Bioconjugated gold nanodots and nanoparticles for protein assays based on photoluminescence quenching. Anal Chem. 2008;80(5):1497-1504.
11. Yuan Z, Peng M, Shi L, et al. Disassembly mediated fluorescence recovery of gold nanodots for selective sulfide sensing. Nanoscale. 2013;5(11):4683-4686.

12. Chen WY, Huang CC, Chen LY, et al. Self-assembly of hybridized ligands on gold nanodots: tunable photoluminescence and sensing of nitrite. Nanoscale. 2014;(19):11078-11083.

13. Huang CC, Chen CT, Shiang YC, et al. Synthesis of fluorescent carbohydrate-protected au nanodots for detection of concanavalin A and Escherichia coli. Anal Chem. 2009;81(3):875-882.

14. Chen WY, Chen LY, Ou CM, et al. Synthesis of fluorescent gold nanodotliposome hybrids for detection of phospholipase $\mathrm{C}$ and its inhibitor. Anal Chem. 2013;85(18):8834-8840.

15. Wei H, Wang Z, Yang L, et al. Lysozyme-stabilized gold fluorescent cluster: synthesis and application as $\mathrm{Hg}^{2+}$ sensor. Analyst. 2010;135(6):1406-1410.

16. Liu Y, Ai K, Cheng X, et al. Gold-nanocluster-based fluorescent sensors for highly sensitive and selective detection of cyanide in water. $A d v$ Funct Mater. 2010;20(6):951-965.

17. Hu L, Han S, Parveen S, et al. Highly sensitive fluorescent detection of trypsin based on BSA-stabilized gold nanoclusters. Biosens Bioelectron. 2012;32(1):297-299.

18. Wang YL, Chen JJ, Irudayaraj J. Nuclear targeting dynamics of gold nanoclusters for enhanced therapy of $\mathrm{HER}^{2+}$ breast cancer. ACS Nano. 2011;5(12):9718-9725.

19. Chen YN, Chen PC, Wang CW, et al. One-Pot synthesis of fluorescent $\mathrm{BSA}-\mathrm{Ce} / \mathrm{Au}$ nanoclusters as ratiometric $\mathrm{pH}$ probes. Chem Commun (Camb). 2014;50(62):8571-8574.

20. Wang CW, Chen YN, Wu BY, et al. Sensitive detection of cyanide using bovine serum albumin-stabilized cerium/gold nanoclusters. Anal Bioanal Chem. 2016;408(1):287-294.

21. Nolf KD, Cosseddu SM, Jasieniak JJ, et al. Binding and packing in twocomponent colloidal quantum dot ligand shells: Linear versus branched carboxylates. J Am Chem Soc. 2017;139(9):3456-3464

22. Roy P, Chen PC, Periasamy AP, et al. Photoluminescent carbon nanodots: Synthesis, physicochemical properties and analytical applications. Materials Today. 2015;18(8):447-458.

23. Chen WY, Chang HY, Lu JK, et al. Self-assembly of antimicrobial peptides on gold nanodots: against multidrug-resistant bacteria and wound healing application. Adv Funct Mater. 2015;25(46):7189-7199. 